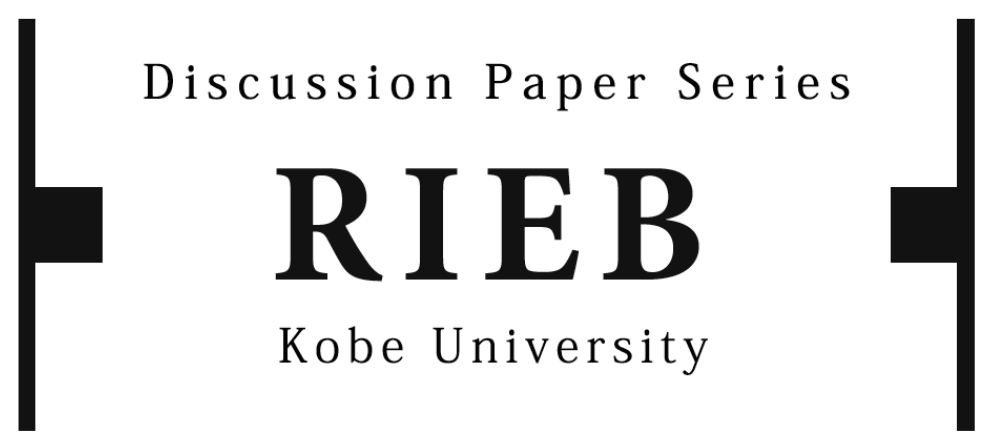

DP2017-27

\title{
Binary Collective Choice with Multiple Premises*
}

RIEB Junior Research Fellow

Masaki MIYASHITA

October 24, 2017

*This Discussion Paper won the Kanematsu Fellowship Prize (FY 2016).

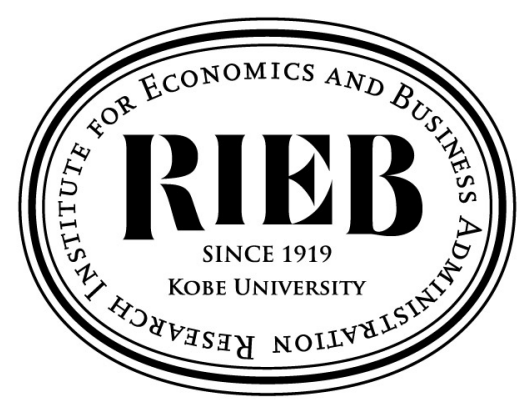

Research Institute for Economics and Business Administration Kobe University 


\title{
Binary Collective Choice with Multiple Premises*
}

\author{
Masaki Miyashita ${ }^{\dagger}$
}

October, 2017

\begin{abstract}
Imagine a group of individuals facing with a complicated yes-no question whose truth value is logically driven from multiple premises. Their purpose is to make a correct group judgment on the question based on their individual judgments. There are two types of ways to aggregate individual judgments: "the premise driven way" (PDW) and "the conclusion driven way" (CDW). We analyze which way is superior to the other to find a correct answer. In our analysis, we introduce a Boolean algebraic approach to formulate a general class of such judgment aggregation problems. We find that if a decision problem is conjunctive, then PDW is more likely to avoid "false acquittance," while CDW is more likely to avoid "false conviction". If a decision problem is disjunctive, the converse of this result holds. These conditions are sufficient to characterize intrinsic biases of aggregation procedures when an aggregation rule possesses no veto power. We also study the asymptotic properties of aggregation procedures, and find that, as the size of a group goes to infinity, PDW ensures the probability that the voting outcome is correct converges to one, while this holds for CDW only if an additional condition is satisfied.
\end{abstract}

Keywords: Social choice, Judgment aggregation, Doctrinal paradox, Condorcet jury theorem, Boolean algebra.

JEL code: D71.

*The Discussion Papers are series of research papers in their draft form, circulated to encourage discussion and comment. Citation and use of such a paper should take account of its provisional character. In some cases, a written consent of the author may be required.

${ }^{\dagger}$ Department of Economics, Yale University; masaki.miyashita@yale.edu; This paper won the Kanematsu Fellowship from the Research Institute for Economics and Business Administration of Kobe University in 2016. I am grateful to Hitoshi Matsushima and Toyotaka Sakai for many suggestions and encouragement, and three anonymous referees of the Kanematsu Fellowship for detailed comments. I would also like to thank Yoko Kawada, Akihiko Matsui, Alan Miller, Yuta Nakamura, Noriaki Okamoto, Daisuke Oyama and Dov Samet for helpful discussions and comments. This paper was presented at Chinese University of Hong Kong, Paris Dauphine University, Ritsumeikan University, Stony Brook University and University of Tokyo, 


\section{Introduction}

In many collective choice situations such as criminal courts, expert panels, boards or committees, a group of individuals faces a complicated question whose answer depends on multiple premises. For example, a criminal court has to decide whether a defendant is guilty or not based on a substantial amount of legal evidence such as the validity of a contract or the presence of bona fide intent. Another example is a decision problem of a personnel committee. Recruiters may agree to hire an applicant as a new member only when he possesses a specialized technique, he has a lot of experience, and he is a good citizen. In such situations, voting is a frequently used method to make a group decision. The recruiters make judgments on the satisfaction of each premise of an applicant, and then their individual judgments are somehow aggregated into a group judgment. However, which answer is derived often crucially depends on which aggregation method is used.

Here is an example of the doctrinal paradox by Kornhauser and Sager (1986). A group of three jurors has to make a decision on whether a defendant is liable for breaching a contract or not (say, conclusion $C$ ). A group decision is made by the majority of the jurors, and the jurors have to make a decision following a legal doctrine. Assume that the fictional doctrine states that a person is liable if and only if he did a certain action (say, first premise $A_{1}$ ) and had contractual obligation not to do that action (say, second premise $A_{2}$ ). This doctrine can be mathematically expressed as a formula $\left(A_{1} \wedge A_{2}\right) \Rightarrow C$. According to a various sort of evidences, each juror makes his own judgment on each proposition $A_{1}$ and $A_{2}$, and his judgment on proposition $C$ follows from the formula $\left(A_{1} \wedge A_{2}\right) \Rightarrow C$, e.g., if a juror considers that $A_{1}$ is true but $A_{2}$ is false, then his conclusion is " $C$ is false". For example, consider the situation given in Table 1.

\begin{tabular}{|c|c|c|c|}
\hline Juror/Proposition & $A_{1}$ & $A_{2}$ & $C$ \\
\hline Juror 1 & True & False & False \\
Juror 2 & False & True & False \\
Juror 3 & True & True & True \\
\hline Majority & True & True & $?$ \\
\hline
\end{tabular}

Table 1: Judgments of jurors

There are two commonly used procedures of using a majority rule to make a group decision. The first procedure is called the premise-driven way (PDW): each juror votes on each premise, and a conclusion follows from the formula $\left(A_{1} \wedge A_{2}\right) \Rightarrow C$. The second procedure is called the conclusiondriven way (CDW): each juror only submits his conclusion to the court. The verdict of a defendant is guilty according to PDW, while he is innocent according to CDW. This contradiction well explains a 
reason why the story is named "doctrinal paradox".

This types of contradictions are known to occur in a variety of contexts. For example, the $O s$ trogorski paradox by Rae and Daubt (1976) displays that indirect voting to parties yields an exactly converse outcome to direct voting on political issues of binary social choice. There is a growing field of judgment aggregation that investigates this type of problems, and many impossibility results have been obtained. ${ }^{1}$

These paradoxical or impossibility results lead us to consider the following question: which is a better way to achieve a correct group decision? This type of analysis dates back to a classical work of Condorcet (1785 [1972]), who has established a mathematical framework of studying voting rules. His main interests were in how to achieve a correct collective choice through voting. Our purpose is to answer this question in the spirit of the Condorcet Jury Theorem (CJT). ${ }^{2}$ We focus on situations in which there are two alternatives on a conclusion-issue (e.g. True or False, Yes or No, $x$ or $y$ ). The correct group decision depends on a state-contingent answer to each premise. However, each individual may make an incorrect decision due to lack of information or rationality. Hence, we investigate which way is better to achieve a "true" conclusion, i.e., find the "truth". 3

In our Theorem 1, we find a necessary and sufficient condition under which one procedure is more likely to avoid "false conviction", or it is less likely to give a false positive judgment in words of statistics. Formally, if the problem with which a group faces is conjunctive, namely, the conclusion is the logical conjunction of the "essential" premises, then PDW accepts the conclusion as long as CDW does so. Also, the converse result holds for the case of a disjunctive decision problem. These two results together establish the characterization of the escape routes from the doctrinal paradox. If in particular, we wish an aggregation rule to have no veto power, PDW and CDW are equivalent if and only if the problem is degenerate, meaning that only one premise dominates all the others; that is, all premises except one are totally neglected in the process of deriving the conclusion. This characterization is interpreted as an impossibility result since such an extreme restriction of premises to be considered is the only way to escape from the doctrinal paradox.

In our Theorem 2, we analyze asymptotic properties of PDW and CDW when the size of a group

\footnotetext{
${ }^{1}$ For example, Baharad, Neeman and Rubinchik (2017), Dietrich (2006, 2007), Dietrich and List (2007, 2008), Dietrich and Mongin (2010), Dokow and Holzman (2010), List and Pettit (2002) and Nehring and Puppe (2008). See also a survey by List and Polak (2010).

${ }^{2}$ In his monograph published in 1785, the Marquis de Condorcet first offered a probabilistic model to analyze voting (Condorcet 1785 [1972]). The Condorcet Jury Theorem shows that when the probability that a single individual makes a correct judgment is higher than 50 percent and all individuals independently make their own judgments, then the probability that the voting outcome is correct converges to $100 \%$ as the size of a group goes to infinity. For technical details and extensions, see Ladha (1992). Condorcet's monograph received almost no attention after his death in the terror era, until Duncan Black's (1985 [2011]) rediscovery of it. Sakai (2015) also explains historical details and conceptual backgrounds.

${ }^{3}$ See Grofman and Feld (1988) and Young (1988).
} 
goes to infinity. Under the usual assumptions of the literature of CJT, using PDW ensures the probability that a majority judgment is correct converges to $100 \%$. That is, an analogue of CJT holds for PDW without any additional assumptions. On the other hand, the usual assumption may not suffice for CDW. In order to establish an analogue of CJT for CDW, the accuracy of an individual judgment must be high when compared with usual situations considered in the literature so far. In the real society, this result would be a reason to support using PDW, or holding a vote issue-by-issue.

There are some papers that compare the truth-tracking properties of PDW and CDW. Grofman (1985) and Bovens and Rabinowicz (2006) obtain a similar conclusion to our finding in Theorem 1. In their analyses, however, the scope of voting rules and regal doctrines to be considered is limited, and the necessity part of our Theorem 1 is only discussed. One of the main differences between our and their analyses is that we give the full characterize of the situations that generate the intrinsic biases of aggregation procedures. Also, our finding in Theorem 1 can be applied without specifying a probability measure which gives a randomness of votes. In fact, the results by Grofman (1985) and Bovens and Rabinowicz (2006) can be obtained as corollaries to our Theorem 1. Bozbay, Dietrich and Peters (2014) and De Clippel and Eliaz (2015) also study the truth-tracking properties of PDW and CDW. They focus on Bayesian strategic voting while our focus is the sincere voting, and different conclusions are derived. ${ }^{4}$

The next section provides a brief note on Boolean lattice, which is needed to state and prove main results. We introduce a model and definitions in Section 3. The first theorem is presented in Section 4, and the second theorem is presented in Section 5. Section 6 concludes our discussion. All omitted proofs are contained in Appendix.

\section{Preliminary}

We introduce some formal lattice theoretic definitions. For detailed explanations, the reader is referred to Gratzer (2002). A partially ordered set (poset) is a pair $(L, \geq)$, where $\geq$ is a reflexive, transitive and antisymmetric binary relation on $L .^{5}$ For $S \subseteq L$, we denote the supremum of $S$ by $\bigvee S$ (referred to as the join of $S$ ) and the infimum by $\bigwedge S$ (referred to as the meet of $S$ ), whenever they exist. A poset $L$ is a lattice if every pair of elements in $L$ has the join and meet. For $a, b \in L$, we write the join of $a$ and $b$ as $a \vee b$, and the meet as $a \wedge b$. A lattice is bounded if it has a greatest element (denoted by $\mathbf{1}$ ) and a least element (denoted by $\mathbf{0}$ ). Every lattice consisting the finite number of elements is

\footnotetext{
${ }^{4}$ In contrast to the study of PDW and CDW for the fixed size of a group, there are a small number of literature which deal with asymptotic properties. Few exceptions include De Clippel and Eliaz (2015).

${ }^{5} \mathrm{~A}$ binary relation $\geq$ is reflexive if for any $a \in L, a \geq a$. It is transitive if for any $a, b, c \in L,[a \geq b$ and $b \geq c]$ implies $a \geq c$. It is antisymmetric if for any $a, b \in L,[a \geq b$ and $b \geq a]$ implies $a=b$.
} 
bounded We can graphically describe finite lattices using the Hasse diagram (see Figure 1 and 2). ${ }^{6}$

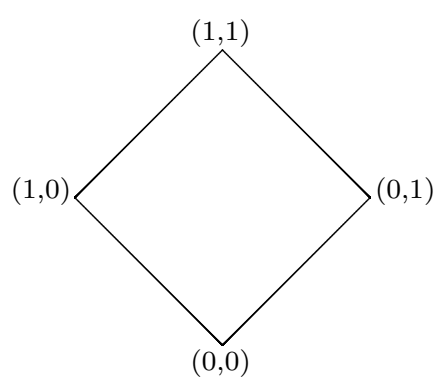

Figure 1: 2-dimensional Boolean lattice

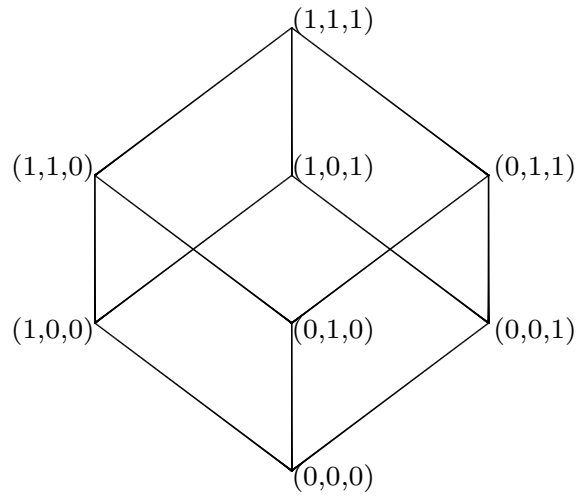

Figure 2: 3-dimensional Boolean lattice

A nonempty subset $K$ of a lattice $L$ is called a sublattice of $L$ if for any $a, b \in K, a \vee b \in S$ and $a \wedge b \in K$ hold. In particular, a sublattice containing $\mathbf{0}$ (resp. $\mathbf{1}$ ) is called a $\{\mathbf{0}\}$-sublattice (resp. $\{\mathbf{1}\}$-sublattice). A sublattice $K$ of $L$ is convex if for any $a, b \in K$ and $c \in L, a<c<b$ implies $c \in K$. Given $a, b \in L$ with $a \leq b$, we define a closed interval by $[a, b] \equiv\{x \in L: a \leq x \leq b\}$. Clearly, $[a, b]$ is a convex sublattice of $L$.

A lattice $L$ is distributive if for any $a, b, c \in L, a \wedge(b \vee c)=(a \wedge b) \vee(a \wedge c)$ and $a \vee(b \wedge c)=(a \vee b) \wedge(a \vee c)$ hold. For $a \in L$, the complement of $a$ is an element $a^{\prime} \in L$ such that $a \vee a^{\prime}=\mathbf{1}$ and $a \wedge a^{\prime}=\mathbf{0}$. A lattice is complemented if every element in $L$ has its complement. A Boolean lattice is a lattice which is distributive and complemented. For example, the set of all binary vectors of length $n$ with the usual component-wise relation $\geq$ (see footnote 6 ) is know as a typical Boolean lattice. In fact, every finite Boolean lattice is isomorphic ${ }^{7}$ to some lattice of binary vectors (see Corollary II.2.12, Gratzer 2002). In this paper, we use the terminology "Boolean lattice" to describe the set of binary vectors. Given a Boolean lattice $B=\{0,1\}^{n}$, the integer $n$ is called the dimension of $B$. For notational convenience, we often write $[n] \equiv\{1, \ldots, n\}$.

A Boolean function is a mapping from a Boolean lattice to $\{0,1\}$. Given a Boolean function $\phi$, we write its domain as $\operatorname{dom}(\phi)$. A Boolean function $\phi$ is nontrivial if it is not constant, and $\phi$ is monotone if for any $z, z^{\prime} \in \operatorname{dom}(\phi), z \geq z^{\prime}$ implies $\phi(z) \geq \phi\left(z^{\prime}\right)$.

\footnotetext{
${ }^{6}$ Each node represents each element. An element $a$ is connected with a higher element $b$ if and only if $b$ covers $a$, i.e., $b>a$ holds and there exists no $c$ such that $b>c>a$. In examples, we consider sets of binary vectors endowed with usual component-wise binary relations. That is, $a \geq b$ if and only if $a_{i} \geq b_{i}$ for any $i \in[n]$.

${ }^{7}$ Two posets $\left(L_{1}, \geq_{1}\right)$ and $\left(L_{2}, \geq_{2}\right)$ are isomorphic if there exists a bijection $f: L_{1} \rightarrow L_{2}$ such that $\left[\forall a, b \in L_{1}, a \geq 1\right.$ $\left.b \Longrightarrow f(a) \geq_{2} f(b)\right]$. Such a function $f$ is called isomorphism. Intuitively, isomorphic two lattices have exactly the same Hasse diagram.
} 
Definition 1 (Conjunctive and Disjunctive). A Boolean function $\phi$ is conjunctive (resp. disjunctive) if there exists some nonempty set $\Delta \subseteq[n]$ and binary vector $c$ of length $|\Delta|$ such that for any $z \in \operatorname{dom}(\phi), \phi(z)=1$ (resp. $=0$ ) if and only if $\left(z_{i}\right)_{i \in \Delta}=c$.

Definition 2 (Degenerate). A Boolean function $\phi$ is degenerate if the value of $\phi$ depends on one and only one component, i.e., there exists some $i \in[n]$ and bijection $\pi:\{0,1\} \rightarrow\{0,1\}$ such that $\phi(z)=\pi\left(z_{i}\right)$ for any $z \in \operatorname{dom}(\phi)$.

Given a Boolean function $\phi$, consider a partition $B_{+}(\phi)$ and $B_{-}(\phi)$ of $d o m(\phi)$ defined by

$$
B_{+}(\phi)=\{z \in \operatorname{dom}(\phi): \phi(z)=1\} \text { and } B_{-}(\phi)=\{z \in \operatorname{dom}(\phi): \phi(z)=0\}
$$

Note that there is a one-to-one correspondence between $\phi$ and $B_{+}(\phi)$, so that several properties of $\phi$ can be restated by the properties of a partition. For example, $\phi$ is nontrivial if and only if $B_{+}(\phi)$ is neither $\emptyset$ nor $\operatorname{dom}(\phi)$, and $\phi$ is monotone if and only if for any $a \in B_{+}(\phi)$ and $b \in \operatorname{dom}(\phi)$ with $b \geq a$, we have $b \in B_{+}(\phi)$. The next lemma gives restatements of Definition 1 and 2 .

Lemma 1. For any Boolean function $\phi$, the following equivalence results hold:

i) $\phi$ is conjunctive if and only if $B_{+}(\phi)$ is a convex sublattice of dom $(\phi)$;

ii) $\phi$ is disjunctive if and only if $B_{-}(\phi)$ is a convex sublattice of dom $(\phi)$;

iii) $\phi$ is degenerate if and only if both $B_{+}(\phi)$ and $B_{-}(\phi)$ are convex sublattices of dom $(\phi)$.

Proof. See Appendix.

Remark. From Lemma 1, only degenerate Boolean functions are those that are both conjunctive and disjunctive.

\section{Model}

\subsection{Decision Problem}

There are a finite number of premise-issues indexed by $1, \ldots, m(m \geq 2)$, and one conclusion-issue. Each issue forms some proposition which takes on truth value 1 (True) or 0 (False). We assume that all premise issues are atomic in the sense that any pair of them are logically independent. The truth value of a conclusion-issue is determined by those of premise-issues via a deduction rule $g$. Formally, $g$ is a Boolean function on $\{0,1\}^{m}$, which maps a vector of evaluations $z=\left(z_{1}, \ldots, z_{m}\right)$ on the premises to an evaluation $g(z)$ on the conclusion. 


\subsection{Individual Judgment}

There are finite numbers of individuals indexed by $1, \ldots, n(n \geq 2)$. Each individual $i \in[n]$ possesses his opinion about the ongoing problem. Let $x_{j}^{i} \in\{0,1\}$ be individual $i$ 's judgment on $j$ th premiseissue, that is, $x_{j}^{i}=1$ (resp. $x_{j}^{i}=0$ ) means that $i$ accepts (resp. rejects) $j$ th premise-issue. A profile of individual judgments is formulated by a matrix form

$$
\boldsymbol{x}=\left(\begin{array}{cccc}
x_{1}^{1} & x_{2}^{1} & \ldots & x_{m}^{1} \\
x_{1}^{2} & x_{2}^{2} & \ldots & x_{m}^{2} \\
\vdots & \vdots & \ddots & \vdots \\
x_{1}^{n} & x_{2}^{n} & \ldots & x_{m}^{n}
\end{array}\right)
$$

We denote $i$ th row of $\boldsymbol{x}$ by $x^{i}=\left(x_{1}^{i}, \ldots, x_{m}^{i}\right)$, interpreted as $i$ 's judgments on all premises. Similarly $j$ th column of $\boldsymbol{x}$ is denoted by $x_{j}=\left(x_{j}^{1}, \ldots, x_{j}^{n}\right)^{\mathrm{T}}$, interpreted as a profile of judgments on $j$ th premise. Let $X_{n} \equiv\{0,1\}^{n \times m}$ be the set of all possible profiles of $n$ individual judgments. Given $x^{i}$, individual $i$ 's judgment on a conclusion-issue is driven by $g\left(x^{i}\right)$. We suppose that there is a consensus on the deduction rule which is in use, i.e., every single individual, as well as a group, follows the same deduction rule to derive his (their) conclusion.

\subsection{Aggregation}

An aggregation rule $f$ is a Boolean function on $\{0,1\}^{n}$, which sums up individual judgments $y=$ $\left(y_{1}, \ldots, y_{n}\right)$ on a certain issue and derives a collective judgment $f(y)$ on that issue. The goal of a group is to make a collective judgment on the conclusion. Given a deduction rule $g$ and an aggregation rule $f$, there are two common ways to obtain a collective judgment on the conclusion.

Definition 3 (PDW). The premise-driven way $P D W_{f, g}$ is a Boolean function on $X_{n}$ defined by $P D W_{g, f}(\boldsymbol{x})=g\left(f\left(x_{1}\right), \ldots, f\left(x_{m}\right)\right)$ for any $\boldsymbol{x} \in X_{n}$.

Definition $4(\mathbf{C D W})$. The conclusion-driven way $C D W_{f, g}$ is a Boolean function on $X_{n}$ defined by $C D W_{g, f}(\boldsymbol{x})=f\left(g\left(x^{1}\right), \ldots, g\left(x^{n}\right)\right)$ for any $\boldsymbol{x} \in X_{n}$.

The premise-driven way (PDW) indirectly aggregates individual judgments, by holding votes on the premises and inferring the collective conclusion via a deduction rule $g$. On the other hand, the conclusion-driven way (CDW) directly aggregates individual judgments on the conclusion, regardless of how individuals make judgments on the premises. 


\section{Judgment Bias}

We are ready to tackle the long standing question relating to the doctrinal paradox: which is a better way to achieve a correct collective judgment, PDW or CDW? Our first theorem characterizes the relations between PDW and CDW in terms of the properties of deduction rules and aggregation rules.

Before we state our theorem, we provide some social choice theoretic interpretations of Boolean functions that are conjunctive, disjunctive or degenerate. Let us recall the judgment aggregation situation in a criminal court. Notice that a deduction rule captures the legal doctrine which connects the answers to the preliminary questions with the verdict of a defendant. If a deduction rule is conjunctive, the regal doctrine requires to prove all "essential" premises indexed by $j \in \Delta$, so as to convict a defendant. In this sense, a conjunctive deduction rule expresses the logical rule of "AND", as the conclusive proposition $C$ is true if and only if the logical conjunction of propositions $\bigwedge_{j \in \Delta} B_{j}$ is true, where $B_{j}$ is either the $j$ th premise $A_{j}$ (if $c_{j}=1$ ) or its negation $\neg A_{j}$ (if $c_{j}=0$ ). Conversely, if a deduction rule is disjunctive, the regal doctrine requires to prove at least one essential premise, so as to convict a defendant. Hence, a disjunctive rule expresses the logical rule of "OR". The interpretation of a degenerate rule might be more obvious: it says there is only one essential premise, or the answer to the conclusion is completely linked with the answer to that premise.

These reads also give us the interpretation for aggregation rules: conjunctive (resp. disjunctive) aggregation rules are non-neutral rules that treat the rejection (resp. acceptance) as the status quo. Moreover, it is worth stressing that the judgments made by individuals outside of $\Delta \subseteq[n]$ are completely ignored in the process of aggregation. That is, an index set $\Delta$ expresses the "influential" voters, or also called "oligarchy". Note that as $\Delta$ shrinks to a singleton set, an aggregation rule collapses into either dictatorial (if $\pi$ is identity) or inverse-dictatorial (otherwise).

Theorem 1. For any nontrivial and monotone $f$ and $g$, the following equivalence results hold:

i) $P D W_{f, g} \geq C D W_{f, g}$ if and only if $g$ is conjunctive or $f$ is disjunctive;

ii) $C D W_{f, g} \geq P D W_{f, g}$ if and only if $g$ is disjunctive or $f$ is conjunctive.

Proof. See Appendix.

An aggregation rule $f$ has no veto power if for any $y \in \operatorname{dom}(f), f(y)=c$ whenever all components of $y$ except one take $c \in\{0,1\}$. Provided that $f$ has no veto power, the relations between PDW and CDW are characterized only by the properties of $g .{ }^{8}$

\footnotetext{
${ }^{8}$ In case $n=2$, any aggregation rule $f$ must have veto power because, otherwise, $f$ cannot determine the value for an input $(0,1)$. Hence, Corollary 1 implicitly assume $n \geq 3$.
} 
Corollary 1. For any monotone $f$ that has no veto power and any nontrivial and monotone $g$, the following equivalence results hold:

i) $P D W_{f, g} \geq C D W_{f, g}$ if and only if $g$ is conjunctive;

ii) $C D W_{f, g} \geq P D W_{f, g}$ if and only if $g$ is disjunctive.

Proof. It suffices to show that if $f$ is either conjunctive or disjunctive, then $f$ must violate the monotonicity or the no veto power condition. Suppose $f$ is conjunctive, and let $\Delta$ and $c$ be as in Definition 1. If $c_{i}=0$ for some $i \in \Delta$, then $f$ is not monotone. So assume $c=(1, \ldots, 1)$. Then fix any $i \in \Delta$, and define $y \in \operatorname{dom}(f)$ by $y_{i}=0$ and $y_{i^{\prime}}=1$ for any $i^{\prime} \neq i$. Since $f$ is conjunctive, this leads $f(y)=0$, which means that an individual $i$ has a veto power on the rejection. The proof in case $f$ is disjunctive is similar.

Here is an example illustrating a situation where two Boolean functions PDW and CDW are incomparable.

Example 1 (Ostrogorski Paradox). Suppose that both $f$ and $g$ are the simple majority rules, so that neither of which are conjunctive and also disjunctive. Consider a collective choice situation with five voters and three premise-issues, interpreted as an election between two political parties labeled by 0 and 1. Each voter compares two parties with respect to three criteria, e.g., diplomacy, welfare service and energy policy, and he would prefer a party with more satisfaction on these criteria. Then let us consider the two profiles given in Table 2, which constitute a dual relationship. From these profiles, it is easily checked that PDW and CDW are incomparable.

\begin{tabular}{|c|c|c|c|c|}
\hline Voter/Proposition & $A_{1}$ & $A_{2}$ & $A_{3}$ & $C$ \\
\hline 1 & 1 & 1 & 0 & 1 \\
2 & 1 & 0 & 1 & 1 \\
3 & 0 & 1 & 1 & 1 \\
4 & 0 & 0 & 0 & 0 \\
5 & 0 & 0 & 0 & 0 \\
\hline Majority & 0 & 0 & 0 & $?$ \\
\hline
\end{tabular}

\begin{tabular}{|c|c|c|c|c|}
\hline Voter/Proposition & $A_{1}$ & $A_{2}$ & $A_{3}$ & $C$ \\
\hline 1 & 0 & 0 & 1 & 0 \\
2 & 0 & 1 & 0 & 0 \\
3 & 1 & 0 & 0 & 0 \\
4 & 1 & 1 & 1 & 1 \\
5 & 1 & 1 & 1 & 1 \\
\hline Majority & 1 & 1 & 1 & $?$ \\
\hline
\end{tabular}

Table 2: Judgments of voters

Combining two statements in Theorem 1, we obtain the next characterization of the escape routes from the doctrinal paradox. Unfortunately, the escape routes are limited: (i) an aggregation rule is dictatorial, (ii) a decision problem reduces to be extremely simple, (iii) the decision making of acceptance is oligarchic, or (iv) the decision making of rejection is oligarchic. 
Corollary 2. For any nontrivial and monotone $f$ and $g, P D W_{f, g}=C D W_{f, g}$ if and only if at least one of the following holds:

i) $f$ is degenerate;

ii) $g$ is degenerate;

iii) Both $f$ and $g$ are conjunctive;

iv) Both $f$ and $g$ are disjunctive.

Proof. By Lemma 1, a Boolean function $\phi$ is degenerate if and only if $\phi$ is both conjunctive and disjunctive. Given this remark, Corollary 2 is a direct consequence of Theorem 1.

Before concluding this section, we claim the robustness of the implications from Theorem 1 in the sense that to compare the probabilities of mistakes, we do not need to specify a probability measure which gives the randomness of votes. That is, any correlations of judgments, among different individuals as well as among different issues, are permitted. Let us consider a probability space $\{\Omega, P\}$, where $\Omega \equiv S \times X_{n}$ and $S \equiv\{0,1\}^{m}$, and $P$ is an arbitrary probability measure on $\Omega .{ }^{9}$ A set $S$ is a state space, whose element specifies the answer to the premises in a true state of nature. Namely, given that $s^{*} \in S$ is a realized state, $s_{j}^{*} \in\{0,1\}$ is the true answer to the $j$ th premise-issue. Suppose that $g$ is a conjunctive deduction rule, and we shall show that CDW avoids a false-positive judgment with a higher probability than PDW does. Fix any $s^{*} \in A$ as a true state of nature, and assume $s^{*} \in B_{-}(g)$. That is, the true verdict of a defendant is "innocent". Then, given some aggregation procedure $F: X_{n} \rightarrow\{0,1\}$, the probability of making a false-positive judgment under $F$ is formulated as $P\left(F(\boldsymbol{x})=1 \mid s^{*}\right)$. Since $g$ is conjunctive, Theorem 1 implies $P D W \geq C D W$, which in turn implies the following set-inclusion relationship:

$$
\left\{\boldsymbol{x} \in X_{n}: C D W(\boldsymbol{x})=1\right\} \subseteq\left\{\boldsymbol{x} \in X_{n}: P D W(\boldsymbol{x})=1\right\}
$$

Hence, by the monotonicity of $P$,

$$
P\left(P D W(\boldsymbol{x})=1 \mid s^{*}\right) \geq P\left(C D W(\boldsymbol{x})=1 \mid s^{*}\right),
$$

showing that PDW is more likely to convict an innocent defendant, when compared to CDW. The strict inequality holds if in addition, $f$ is not conjunctive, $g$ is not degenerate, and the conditional

\footnotetext{
${ }^{9}$ Since $\Omega$ is a finite set, we employ the power set of it as the underlying $\sigma$-field.
} 
probability $P\left(\cdot \mid s^{*}\right)$ has a full domain on $X_{n}$. In a similar fashion, CDW is shown to be more likely to acquit a guilty defendant than CDW.

These argument imply that there is a trade-off between avoiding false-conviction and false-accuitance. If we wish to use an aggregation procedure that is effective to avoid false conviction, we need to give up the performance of an aggregation procedure to detect a guilty defendant to some extent. In practical use, the ratio of the potential social costs from false positive and negative judgments are critical for the choice of aggregation procedures to be used.

\section{$5 \quad$ Asymptotic Property}

The existence of judgment biases are discussed in the previous section. In this section, we develop our discussion by examining the degree of judgment biases that each aggregation procedure possesses. Our second theorem shows that the drawback of PDW vanishes as the number of individuals increases, whereas that of CDW may not. More precisely, the theorem provides a sufficient condition under which an analogue of the Condorcet Jury Theorem (CJT) holds for each aggregation procedure.

CJT is a cornerstone of social choice theory, which provides some justification for using the simple majority rule. It shows that if individual judgments are drawn from i.i.d. Bernoulli distribution with a success probability lager than a half, then the probability that the majority judgment is correct converges to one as the size of a group goes to infinity. Formally, it is formulated as follows:

Condorcet Jury Theorem. (Condorcet 1785 [1972], Black 1958 [2011], Ladha 1992) Suppose that there are two alternatives " $C$ " (correct) and " $D$ " (incorrect), and each individual $i \in[n]$ votes for $x_{i} \in\{C, D\}$, where $x_{i}$ follows a Bernoulli distribution with a success probability $v>\frac{1}{2}$. If any $x_{i}$ and $x_{j}(i \neq j)$ are statistically independent, then

$$
\operatorname{Pr}\left(\left|i \in[n]: x_{i}=C\right| \geq \frac{n}{2}\right) \rightarrow 1 \text { as } n \rightarrow \infty \text {. }
$$

To study asymptotic properties, we slightly modify our model. Let $\mathbb{N}$ be the set of all integers, which describe potential populations. An aggregation rule $f: \bigcup_{n=1}^{\infty}\{0,1\}^{n} \rightarrow\{0,1\}$ is a function which sums up judgments of turnout individuals. In this section we focus on the simple majority rule with an appropriate tie-breaking condition. The domains of PDW and CDW are similarly extended to be $\bigcup_{n=1}^{\infty} X_{n}$. The group decision problem is taken place on the family of probability space $\left\{\left(\Omega_{n}, P_{n}\right)\right\}_{n \in \mathbb{N}}$, where $\Omega_{n} \equiv S \times X_{n}$. We specify our probability measures so that they become multi-dimensional analogoues of the one in the jury theorem: suppose that $\left\{P_{n}\right\}_{n \in \mathbb{N}}$ is a family of probability measures 
on $\left\{\Omega_{n}\right\}_{n \in \mathbb{N}}$ such that

i) For any $n, n^{\prime} \in \mathbb{N}, P_{n}(s)=P_{n^{\prime}}(s)>0$;

ii) For any $n \in \mathbb{N}$ and any $(i, j) \in[n] \times[m], P_{n}\left(x_{j}^{i}=s_{j} \mid s\right)=v$, where $\frac{1}{2}<v<1$;

iii) For any $n \in \mathbb{N}$ and any $(i, j),\left(i^{\prime}, j^{\prime}\right) \in[n] \times[m]$ with $(i, j) \neq\left(i^{\prime}, j^{\prime}\right), x_{i}^{j}$ and $x_{j^{\prime}}^{i^{\prime}}$ are stochastically independent, that is, for any $c, c^{\prime} \in\{0,1\}$,

$$
P_{n}\left(x_{j}^{i}=c \text { and } x_{j^{\prime}}^{i^{\prime}}=c^{\prime} \mid s\right)=P_{n}\left(x_{j}^{i}=c \mid s\right) \cdot P_{n}\left(x_{j^{\prime}}^{i^{\prime}}=c^{\prime} \mid s\right)
$$

The realization of a state $s^{*} \in S$ specifies the true answer to each premise, and accordingly, the true answer to the conclusion is determined by $g\left(s^{*}\right)$. The goal of individuals is to achieve $g\left(s^{*}\right)$ as a consequence of voting. However, a single individual may make a wrong choice because of lack of information or rationality. The condition (i) is an auxiliary assumption arguing that the marginal distribution of $s$ has a full domain on $S$ and is independent from the number of turnout individuals. The condition (ii) asserts a single individual can achieve a true answer of each premise by chance $v$, which is higher than a half. This assumption is usual in the literature as it means reasoning of a single individual is at least superior to flipping a fair coin. The condition (iii) is an independence condition, which include both the "inter-personal independence" (different individuals judge independently) and "intra-personal independences" (different issues are judged independently). Following the above specification of $P_{n}$, the conditional probability of drawing a profile $\boldsymbol{x} \in X_{n}$, given a state realization $s^{*} \in S$, is formulated as

$$
P_{n}\left(\boldsymbol{x} \mid s^{*}\right)=\prod_{j=1}^{m} v^{\left|\left\{i \in N: x_{j}^{i}=s_{j}^{*}\right\}\right|} \cdot(1-v)^{\left|\left\{i \in N: x_{j}^{i} \neq s_{j}^{*}\right\}\right|} .
$$

Before we state the second theorem, let us introduce some definitions. Given $v \in\left(0, \frac{1}{2}\right)$, define a function $v_{g}(v): S \rightarrow[0,1]$ by $v_{g}(v)(s) \equiv P_{n}\left(g\left(x_{i}\right)=g(s) \mid s\right)$ for each $s \in S$. This is a conditional probability that a single individual achieves the correct conclusion, conditioning on a true state to be $s \in S .{ }^{10}$ Let $v_{g}(v) \equiv \min _{s \in S} v_{g}(v)(s)$, and call this a competence value. A competence value expresses the confidence in one's opinion on the conclusion. This value plays a decisive role for the asymptotic property of CDW. Now, we are ready to state our second theorem.

\footnotetext{
${ }^{10}$ By the specification of the probability measures, $v_{g}(v)(\cdot)$ is shown to be independent from $n$ and $i$.
} 
Theorem 2. Let $f$ be the simple majority rule. For any nontrivial deduction rule $g$,

i) $\lim _{n \rightarrow \infty} P_{n}(P D W$ is correct $)=1$;

ii) $\lim _{n \rightarrow \infty} P_{n}(C D W$ is correct $)=1$ if and only if $v_{g}(v)>\frac{1}{2}$, and $v_{g}(v)$ turns out to be

$$
v_{g}(v)=\min _{s \in S} \sum_{s^{\prime}: g\left(s^{\prime}\right)=g(s)} v^{m-\left\|s, s^{\prime}\right\|} \cdot(1-v)^{\left\|s, s^{\prime}\right\|}
$$

where $\|\cdot\|$ denotes the Hamming distance on $S$.

Proof. See Appendix.

Theorem 2 provides some conditions for PDW and CDW to establish the analogue of CJT. The asymptotic property of PDW does not require no more than that $v$ is larger than a half. 11 On the other hand, the asymptotic property of CDW crucially depends on a competence value. The next lemma gives some evaluations about this condition.

Lemma 2. If $g$ is either conjunctive or disjunctive, then $v_{g}(v)=v^{|\Delta|}$, where $|\Delta|$ is the number of essential premise.

Proof. See Appendix.

Hence, when $g$ is either conjunctive or disjunctive, $v_{g}(v)$ is strictly less than $v$ unless $g$ is degenerate, which corresponds to the case $|\Delta|=1$. This observation supports the merit of PDW as we know CDW may require a harder condition than PDW. Although the validity of Theorem 2 seems to depend on the specification of the probability measures, the basic idea underlying here is analogous to the multiple comparison in statistic models. That is, even if each individual can achieve the true judgment on each premise, it is difficult to answer a number of premises simultaneously and derive the true conclusion. In general, it is difficult to compute a competence value in a direct manner, unless $g$ is either conjunctive or disjunctive. With regard to this point, let us leave the following conjecture as an open question.

Conjecture. For any deduction rule $g$ and any $v \in\left(\frac{1}{2}, 1\right)$, we have $v_{g}(v) \leq v$, where the equality holds if and only if $g$ is degenerate.

\footnotetext{
${ }^{11}$ To have $v$ larger than a half is sufficient for statement (i). In fact, this is a necessary and sufficient condition for a group to reach the true conclusion for right reason, whereas the collective conclusion might coincide with the truth for a wrong reason. For example, assume a deduction rule is disjunctive and there are two premises. If a true state agrees one and only one of them, even when the majority of individuals makes mistakes on both premises, a group can rightly reject conclusion. If we consider such possibilities of achieving the true conclusion for wrong reason, a milder condition is enough for PDW.
} 


\section{Conclusion}

We have studied binary social choice problems such that the conclusion is logically connected with the premises. We have compared two commonly used aggregation procedures called PDW and CDW. When the logical connection has a simple form such as conjunctive or disjunctive ones, one procedure is always biased toward a certain direction. This result is suggestive for criminal courts which wishes to avoid false conviction. For example, when the legal doctrine requires proving multiple premises for the conviction, we can recommend a criminal court to use CDW in order to avoid a false positive judgment with higher probability. However, in other situations where the false positive and negative judgments potentially induce the same amount of the social cost, PDW is recommended to use as it exploits more benefits from the wisdom of the crowd.

\section{Appendix A: Proofs}

\section{Lemma 1}

Proof of (i) and (ii). Suppose $\phi$ is conjunctive. Set $\underline{z} \in \operatorname{dom}(\phi)$ to be $\left(\underline{z}_{i}\right)_{i \in \Delta}=d$ and $\left(\underline{z}_{i}\right)_{i \notin \Delta}=$ $(0, \ldots, 0)$. Similarly, set $\bar{z} \in \operatorname{dom}(\phi)$ to be $\left(\bar{z}_{i}\right)_{i \in \Delta}=d$ and $\left(\bar{z}_{i}\right)_{i \notin \Delta}=(1, \ldots, 1)$. Since $\Delta \neq \emptyset$, we have $\underline{z} \neq \bar{z}$. Then, obviously $B_{+}(\phi)=[\underline{z}, \bar{z}]$, which is a convex sublattice of $\operatorname{dom}(\phi)$.

Conversely, suppose $B_{+}(\phi)$ is a convex sublattice of $\operatorname{dom}(\phi)$. Since $B_{+}(\phi)$ is finite, iteratively applying the property of sublattice, we have $\wedge B_{+}(\phi)=\left(z_{k} \wedge \cdots\left(z_{3} \wedge\left(z_{1} \wedge z_{2}\right)\right)\right) \in B_{+}(\phi)$. Similarly, we have $\bigvee B_{+}(\phi) \in B_{+}(\phi)$. Let $\underline{z}=\bigwedge B_{+}(\phi)$ and $\bar{z}=\bigvee B_{+}(\phi)$. Then, by the convexity of $B_{+}(\phi)$, we have $B_{+}(\phi)=[\underline{z}, \bar{z}]$. Define $\Delta \equiv\left\{i \in[n]: \underline{z}_{i}=\bar{z}_{i}\right\}$. Observe that for any $z \in \operatorname{dom}(\phi)$,

$$
\phi(z)=1 \Longleftrightarrow z \in[\underline{z}, \bar{z}] \Longleftrightarrow\left(z_{i}\right)_{i \in \Delta}=\left(\bar{z}_{i}\right)_{i \in \Delta},
$$

which shows that $\phi$ is conjunctive. Hence, we proved (i). The proof of (ii) is similar.

Proof of (iii). Suppose $\phi$ is degenerate. Let $i \in[n]$ be an index as in Definition 2. Then observe that

$$
B_{+}(\phi)=\left\{z \in \operatorname{dom}(\phi): z_{i}=\pi^{-1}(1)\right\} \text { and } B_{-}(\phi)=\left\{z \in \operatorname{dom}(\phi): z_{i}=\pi^{-1}(0)\right\}
$$

which imply that $\phi$ is conjunctive and disjunctive. Hence, by (i) and (ii), both $B_{+}(\phi)$ and $B_{-}(\phi)$ are convex sublattices of $\operatorname{dom}(\phi)$.

Conversely, suppose both $B_{+}(\phi)$ and $B_{-}(\phi)$ are convex sublattices of $\operatorname{dom}(\phi)$. By (i) and (ii), 
there are some nonempty subsets $\Delta_{+}$and $\Delta_{-}$of $[\mathrm{n}]$, and binary vectors $c^{+}$and $c^{-}$such that

$$
B_{+}(\phi)=\left\{z \in \operatorname{dom}(\phi):\left(z_{i}\right)_{i \in \Delta_{+}}=c^{+}\right\} \text {and } B_{-}(\phi)=\left\{z \in \operatorname{dom}(\phi):\left(z_{i}\right)_{i \in \Delta_{-}}=c^{-}\right\}
$$

First, we shall show that $\Delta_{+}=\Delta_{-}$. Suppose not, without loss of generality, there exists $i \in[n]$ such that $i \in \Delta_{+}$and $i \notin \Delta_{-}$. Then for $z \in \operatorname{dom}(\phi)$ with $z_{i} \neq c_{i}^{+}$and $z_{j} \neq c_{j}^{-}$for any $j \in \Delta_{-}$, neither $z \in B_{+}(\phi)$ nor $z \in B_{-}(\phi)$ hold. This is a contradiction to that $B_{+}(\phi)$ and $B_{-}(\phi)$ partition dom $(\phi)$. Thus, henceforth, we denote $\Delta=\Delta_{+}=\Delta_{-}$.

Second, we claim that $\Delta$ is a singleton. Note that, by (1), the cardinalities of $B_{+}(\phi)$ and $B_{-}(\phi)$ are $2^{n-|\Delta|}$. Since $B_{+}(\phi)$ and $B_{-}(\phi)$ partition $\operatorname{dom}(\phi)$, whose cardinality is $2^{n}$,

$$
2^{n-|\Delta|}+2^{n-|\Delta|}=2^{n}
$$

which in turn implies

$$
2^{1-|\Delta|}=1
$$

Solving this equation, we get $|\Delta|=1$. Hence, $c^{+}$and $c^{-}$are numbers.

Finally, if $c^{+}=c^{-}$, then $B_{+}(\phi)=B_{-}(\phi)$, which contradicts that they are disjoint. Hence, we must have $c^{+} \neq c^{-}$. Then a function $\pi:\{0,1\} \rightarrow\{0,1\}$ defined by $\pi(1)=c^{+}$and $\pi(0)=c^{-}$is bijective. Substituting $\pi(\cdot)$ and $\Delta=\Delta_{+}=\Delta_{-}$into (1), we conclude that $\phi$ is degenerate.

\section{Theorem 1}

It suffices to show only (i) because (ii) follows from the lattice dual argument. Note that since $P D W_{f, g}$ and $C D W_{f, g}$ are Boolean functions, $P D W_{f, g} \geq C D W_{f, g}$ is equivalent to that $\left[\forall \boldsymbol{x} \in X_{n}, C D W_{f, g}(\boldsymbol{x})=\right.$ $\left.1 \Rightarrow P D W_{f, g}(\boldsymbol{x})=1\right]$, which is also equivalent to saying that $\left[\forall \boldsymbol{x} \in X_{n}, P D W_{f, g}(\boldsymbol{x})=0 \Rightarrow\right.$ $\left.C D W_{f, g}(\boldsymbol{x})=0\right]$.

If part of (i). Suppose that $g$ is conjunctive. Then, by definition, there exist some nonempty set $\Delta \subseteq[m]$ and binary vector $c$ of length $|\Delta|$ such that

$$
\forall z \in \operatorname{dom}(g), g(z)=1 \Longleftrightarrow\left(z_{j}\right)_{j \in \Delta}=c
$$

In particular, the monotonicity of $f$ implies $c=(1, \ldots, 1)$. Take any $\boldsymbol{x} \in X_{n}$ and assume $C D W_{f, g}(\boldsymbol{x})=$ 1 , that is, $f\left(g\left(x^{1}\right), \ldots, g\left(x^{n}\right)\right)=1$. Let us show that

$$
\forall j \in \Delta, x_{j} \geq\left(g\left(x^{1}\right), \ldots, g\left(x^{n}\right)\right) .
$$


Indeed, for any $j \in \Delta$ and any $i \in[n], g\left(x^{i}\right)=1$ and (2) together imply $x_{j}^{i}=1$. Hence, by (3) and the monotonicity of $f, f\left(x_{j}\right)=1$ for any $j \in \Delta$. Thus, by (2), $P D W_{f, g}(\boldsymbol{x})=g\left(f\left(x_{1}\right), \ldots, f\left(x_{m}\right)\right)=1$, as desired.

The proof in case that $f$ is disjunctive is similar, but for the sake of completion, we repeat the same steps. If $f$ is disjunctive, then there exist some nonempty $\Delta \subseteq[n]$ such that

$$
\forall y \in \operatorname{dom}(f), f(y)=0 \Longleftrightarrow\left(y_{i}\right)_{i \in \Delta}=(0, \ldots, 0)
$$

Take any $\boldsymbol{x} \in X_{n}$ and assume $P D W_{f, g}(\boldsymbol{x})=g\left(f\left(x_{1}\right), \ldots, f\left(x_{m}\right)\right)=0$. We claim that

$$
\forall i \in \Delta, x^{i} \leq\left(f\left(x_{1}\right), \ldots, f\left(x_{m}\right)\right)
$$

Indeed, for any $i \in \Delta$ and any $j \in[m], f\left(x_{j}\right)=0$ and (4) together imply $x_{j}^{i}=0$. Hence, by (5) and the monotonicity of $g, g\left(x^{i}\right)=0$ for any $i \in \Delta$. Therefore, by (4), $C D W_{g, f}(\boldsymbol{x})=f\left(g\left(x^{1}\right), \ldots, g\left(x^{n}\right)\right)=0$, as desired.

Only if part of (i). We use a contraposition argument. By Lemma 1 (i), if $g$ is not conjunctive, then $B_{+}(g)$ is not a convex sublattice of $\operatorname{dom}(g)$. Then there are the following three potential cases:

(a) $\exists z, z^{\prime} \in B_{+}(g)$ s.t. $z \wedge z^{\prime} \notin B_{+}(g)$;

(b) $\exists z, z^{\prime} \in B_{+}(g)$ s.t. $z \vee z^{\prime} \notin B_{+}(g)$;

(c) $\exists z, z^{\prime} \in B_{+}(g), \exists z^{\prime \prime} \in \operatorname{dom}(g)$ with $z>z^{\prime \prime}>z^{\prime}$ s.t. $z^{\prime \prime} \notin B_{+}(g)$.

However, by monotonicity of $g,(\mathrm{~b})$ and (c) do not occur. Hence, there exist two distinct elements $z$ and $z^{\prime}$ in $B_{+}(g)$ such that $z \wedge z^{\prime} \notin B_{+}(g)$.

Similarly, by Lemma 1 (ii), if $f$ is not disjunctive, then $B_{-}(f)$ is not a convex sublattice of $\operatorname{dom}(f)$. Then, again by the monotonicity of $f$, there are two distinct elements $y, y^{\prime} \in B_{-}(f)$ such that $y \vee y^{\prime} \notin$ $B_{-}(f)$. Define subsets of individuals $U$ and $T$ by $U \equiv\left\{i \in[n]: y_{i}=1\right\}$ and $T \equiv\left\{i \in[n]:\left(y \vee y^{\prime}\right)_{i}=1\right\}$. Since $y \vee y^{\prime} \neq y$, we have $y \vee y^{\prime}>y$, which in turn implies $U \subsetneq T$. Thus, $U^{\prime} \equiv T \backslash U$ is a nonempty set of individuals. In addition, if $y=\mathbf{0}$, then $y \vee y^{\prime}=y^{\prime} \in B_{-}(f)$, which leads a contradiction. Hence, there is at least one $i \in[n]$ with $y_{i}=1$, and thus $U$ is also nonempty. Then, define profile $\boldsymbol{x} \in X^{n}$ as described in Table 3.

All individuals belonging to $U$ submit judgment profile $z$, and those belonging to $U^{\prime}$ submit $z^{\prime}$. Note that since $z, z^{\prime} \in B_{+}(g)$, individuals in $T=U \cup U^{\prime}$ ascent to the conclusion. The individuals 


\begin{tabular}{c|ccc|c} 
Voter/Issue & $A_{1}$ & $\cdots$ & $A_{m}$ & $C$ \\
\hline$U$ & $z_{1}$ & $\cdots$ & $z_{m}$ & 1 \\
$U^{\prime}$ & $z_{1}^{\prime}$ & $\cdots$ & $z_{m}^{\prime}$ & 1 \\
$N \backslash T$ & 0 & $\cdots$ & 0 & 0 \\
\hline & $f\left(x_{1}\right)$ & $\cdots$ & $f\left(x_{m}\right)$ &
\end{tabular}

Table 3: The construction of profile $\boldsymbol{x}$

in $N \backslash T$ descent to all premises, and they also descent to the conclusion by the non-triviality and monotonicity of $g$. Hence, by the construction of $T$, we have $C D W_{f, g}(\boldsymbol{x})=1$.

Let us show that $\left(f\left(x_{1}\right), \ldots, f\left(x_{m}\right)\right)=z \wedge z^{\prime}$. To this end, fix any $j \in[m]$. If $\left(z \wedge z^{\prime}\right)_{j}=1$ then $z_{j}=z_{j}^{\prime}=1$, and thus $x_{j}=y \vee y^{\prime}$. Hence, by $y \vee y^{\prime} \in B_{+}(f)$, we have $f\left(x_{j}\right)=1$. Conversely, $\left(z \wedge z^{\prime}\right)_{j}=0$ implies that either $z_{j}$ or $z_{j}^{\prime}$ takes 0 . If $z_{j}^{\prime}=0$, then $x_{j}$ equals either $\mathbf{0}$ or $y$, so that $x_{j} \leq y$. Otherwise, provided that $U^{\prime}=T \backslash U \subseteq\left\{i \in[n]: y_{i}^{\prime}=1\right\}$, we have $x_{j} \leq y^{\prime}$. In any case, since $y, y^{\prime} \in B_{-}(f)$ and $f$ is monotone, we get $f\left(x_{j}\right)=0$. Therefore, $\left(f\left(x_{1}\right), \ldots, f\left(x_{m}\right)\right)=z \wedge z^{\prime}$. Hence, by $z \wedge z^{\prime} \in B_{-}(g)$, we obtain $P D W_{f, g}(\boldsymbol{x})=0$.

\section{Theorem 2}

Given an aggregation procedure $F$, the total probability formula yields

$$
P_{n}(F \text { is correct })=\sum_{s^{*} \in S} P_{n}(s) \cdot P_{n}\left(F \text { is correct } \mid s^{*}\right) .
$$

Since we suppose the marginal of $P_{n}$ on $S$ is independent from $n$ and has a full domain, it suffices to show that the conditional probability $P_{n}(F$ is correct $\mid s)$ converges to 1 for any $s \in S$. So, we proceeds the proof by fixing any $s^{*} \in S$ as a realized state.

Asymptotic property of PDW. Observe the following equality holds:

$$
\begin{aligned}
P_{n}\left(\mathrm{PDW} \text { is correct } \mid s^{*}\right) & =P_{n}\left(g\left(f\left(x_{1}\right), \ldots, f\left(x_{n}\right)\right)=g\left(s^{*}\right) \mid s^{*}\right) \\
& \geq P_{n}\left(f\left(x_{1}\right)=s_{1}^{*}, \ldots, f\left(x_{m}\right)=s_{m}^{*} \mid s^{*}\right),
\end{aligned}
$$

which means a group achieves the true conclusion whenever they answer all premises correctly. By 
the independence condition of $P_{n}$,

$$
P_{n}\left(f\left(x_{1}\right)=s_{1}^{*}, \ldots, f\left(x_{m}\right)=s_{m}^{*} \mid s^{*}\right)=\prod_{j=1}^{m} P_{n}\left(f\left(x_{j}\right)=s_{j}^{*} \mid s^{*}\right) .
$$

Note that $x_{j}^{i}(i=1, \ldots, n)$ is independently drawn from the Bernoulli distribution. Thus, by the Condorcet Jury Theorem, each $P_{n}\left(f\left(x_{j}\right)=s_{j}^{*} \mid s^{*}\right)$ converges to 1 as $n \rightarrow \infty$. Hence, by (6) and (7), we obtain $\lim _{n \rightarrow \infty} P_{n}\left(\mathrm{PDW}\right.$ is correct $\left.\mid s^{*}\right)=1^{m}=1$.

Asymptotic property of CDW. Observe that

$$
P_{n}\left(\mathrm{CDW} \text { is correct } \mid s^{*}\right)=P_{n}\left(f\left(g\left(x^{1}\right), \ldots, g\left(x^{n}\right)\right)=g\left(s^{*}\right) \mid s^{*}\right) .
$$

Given $s^{*} \in S$, each $g\left(x^{i}\right)$ is viewed as a binary random variable which takes $g\left(s^{*}\right)$ with probability $v_{g}(v)\left(s^{*}\right)$. By the independence condition, $g\left(x^{i}\right)$ is stochastically independent $(i=1, \ldots, n)$. Thus, the Condorcet Jury Theorem is applicable for the voting on a conclusion-issue, and it yields that $P_{n}\left(\mathrm{PDW}\right.$ is correct $\left.\mid s^{*}\right)$ converges to 1 if and only if $v_{g}(v)\left(s^{*}\right)>\frac{1}{2}$. Since $P_{n}$ has a full domain on $S$,

$$
v_{g}(v)=\min _{s \in S} v_{g}(v)(s)>\frac{1}{2}
$$

is a necessary and sufficient condition for the asymptotic property of CDW. We shall show that $v_{g}(v)$ is expressed as in Theorem 2. Consider the event that an individual $i$ holds a jusgment $s \in S$ in the state $s^{*}$. By the independence condition, the probability of this event turns out

$$
P_{n}\left(x^{i}=s \mid s^{*}\right)=v^{m-\left\|s^{*}, s\right\|} \cdot(1-v)^{\left\|s^{*}, s\right\|}
$$

Summing this formula over all jusgments which give the same conclusion as $s^{*}$,

$$
v_{g}(v)\left(s^{*}\right)=\sum_{s: g(s)=g\left(s^{*}\right)} v^{m-\left\|s^{*}, s\right\|} \cdot(1-v)^{\left\|s^{*}, s\right\|} .
$$

Hence, a competence value takes the form as in Theorem 2 . 


\section{Lemma 2}

Suppose $g$ is conjunctive. By Lemma $1, B_{+}(g)$ is a convex sublattice of $\operatorname{dom}(g)$ that has the following representation with some nonempty $\Delta \subseteq[m]$ and binary vector $c$ of length $|\Delta|$

$$
B_{+}(g)=\left\{s \in \operatorname{dom}(g):\left(s_{j}\right)_{j \in \Delta}=c\right\}
$$

Let $k \equiv m-|\Delta|$. Note that $B_{+}(g)$ is isomorphic to $k$-dimensional Boolean lattice. Hence, for each $s^{+} \in B_{+}(g)$ and each $j=0,1, \ldots, k$, there are ${ }_{k} C_{j}$ number of $s \in B_{+}(g)$ such that $\left\|s^{+}, s\right\|=j$. Therefore, by applying the binomial theorem, for any $s^{+} \in B_{+}(g)$,

$$
\begin{aligned}
v_{g}(v)\left(s^{+}\right) & =\sum_{s \in B_{+}(g)} v^{m-\left\|s^{+}, s\right\|} \cdot(1-v)^{\left\|s^{+}, s\right\|} \\
& =v^{m-k} \sum_{j=0}^{k}{ }_{k} C_{j} v^{k} \cdot v^{j}=v^{m-k}(v-(1-v))^{k}=v^{|\Delta|} .
\end{aligned}
$$

Take any $s^{-} \in B_{-}(g)$. Then, $s_{j}^{-} \neq c_{j}$ for some $j \in \Delta$. Let $A \equiv\left\{s \in \operatorname{dom}(g): s_{j} \neq c_{j}\right\}$. By the construction, $A$ is a convex sublattice containing $s^{-}$, and isomorphic to $(m-1)$-dimensional Boolean lattice. Moreover, $A \cap B_{+}(g)=\emptyset$, and thus $A \subseteq B_{-}(g)$. Hence, again by the binomial theorem,

$$
\begin{aligned}
v_{g}(v)\left(s^{-}\right) & =\sum_{s \in B_{-}(g)} v^{m-\left\|s^{-}, s\right\|}(1-v)^{\left\|s^{-}, s\right\|} \\
& \geq \sum_{s \in A} v^{m-\left\|s^{-}, s\right\|}(1-v)^{\left\|s^{-}, s\right\|}=v .
\end{aligned}
$$

This equation, together with $|\Delta| \geq 1$ and $v \in\left(\frac{1}{2}, 1\right)$, yields $v_{g}(v)\left(s^{-}\right) \geq v^{|\Delta|}$. Therefore, $v_{g}(v)=$ $\min _{s \in S} v_{g}(v)(s)=v^{|\Delta|}$. The proof in case $g$ is disjunctive is similar. 


\section{References}

Baharad, E., Neeman, Z. and Rubinchik, A. (2017) "Aggregation of Opinions is Hard," mimeo.

Black, D. (1958 [2011]) The Theory of Committees and Elections, reprinted by Cambridge University Press.

Bovens, L. and Rabinowicz, W. (2006) "Democratic Answers to Complex Questions - An Epistemic Perspective," Synthese, Vol. 150, pp. 131-153.

Bozbay, I., Dietrich, F. and Peters, H. (2014) "Judgment Aggregation in Search for the Truth," Games and Economic Behavior, Vol. 87, pp. 571-590.

Condorcet, M. de (1785 [1972]) Essau sur l'application de l'analyse à la probabilité des décisions rendues à la pluralité des voix, reprinted by Chelfea Publishing Company.

De Clippel, G. and Eliaz, K. (2015) "Premise-Based Versus Outcome-Based Information Aggregation," Games and Economic Behavior, Vol. 89, pp. 34-42.

Dietrich, F. (2006) "Judgment Aggregation: (im)possibility theorems," Journal of Economic Theory, Vol. 126, pp.286-298.

Dietrich, F. (2007) "A Generalized Model of Judgment Aggregation," Social Choice and Welfare, Vol. 28, pp. 529-565.

Dietrich, F. and List, C. (2007) "Arrow's Theorem in Judgment Aggregation," Social Choice and Welfare, Vol. 29, pp. 19-33.

Dietrich, F. and List, C. (2008) "A Liberal Paradox for Judgment Aggregation," Social Choice and Welfare, Vol. 31, pp. 59-78.

Dietrich, F. and Mongin, P. (2010) "The Premise-base Approach to Judgment Aggregation," Journal of Economic Theory, Vol. 145, pp. 562-582.

Dokow, E. and Holzman, R. (2010) "Aggregation of Binary Evaluations," Journal of Economic Theory, Vol. 145, pp. 495-511.

Gratzer, G. (2002) General Lattice Theory, Springer Science \& Business Media.

Grofman, B. (1985) "Research Note: The Accuracy of Group Majorities for Disjunctive and Conjunctive Decision Tasks," Organizational Behavior and Human Decision Processes, Vol. 35, pp. 119-123. 
Grofman, B. and Feld, S. L. (1988) "Rousseau's General Will: A Condorcetian Perspective," American Political Science Review, Vol. 82, pp. 567-576.

Kornhauser, L. A. and Sager, L. G. (1986) "Unpacking the Court," Yale Law Journal, Vol. 96, pp. 82-117.

Ladha, K. (1992) "The Condorcet Jury Theorem, Free Speech, and Correlated Votes," American Journal of Political Science, Vol. 36, pp. 617-634.

List, C. and Pettit, P. (2002) "Aggregating Sets of Judgments: An Impossibility Results," Economics and Philosophy, Vol. 18, pp. 89-110.

List, C. and Polak, B. (2010) "Introduction to Judgment Aggregation," Journal of Economic Theory, Vol. 145, pp. 441-466.

Nehring, K. and Puppe, C. (2008) "Consistent Judgment Aggregation: The True-functional Case," Social Choice and Welfare, Vol. 31, pp. 41-57.

Rae, D. W. and Daudt, H. (1976) "The Ostrogorski Paradox: A Peculiarity of Compound Majority Decision," European Journal of Political Research, Vol. 4, pp. 391-398.

Sakai, T. (2015) "Considering Collective Choice: The Route 328 Problem in Kodaira City," Japanese Economic Review, forthcoming.

Young, H. P. (1988) "Condorcet's Theory of Voting," American Political Science Review, Vol 82, pp. 1231-1244. 